Research Article

\title{
Global Bifurcation of Positive Solutions of Asymptotically Linear Elliptic Problems
}

\author{
Ruyun Ma, Yanqiong Lu, and Ruipeng Chen \\ Department of Mathematics, Northwest Normal University, Lanzhou 730070, China \\ Correspondence should be addressed to Ruyun Ma; ruyun_ma@126.com \\ Received 13 March 2014; Revised 25 April 2014; Accepted 8 May 2014; Published 25 May 2014 \\ Academic Editor: Gennaro Infante
}

Copyright (c) 2014 Ruyun Ma et al. This is an open access article distributed under the Creative Commons Attribution License, which permits unrestricted use, distribution, and reproduction in any medium, provided the original work is properly cited.

We are concerned with determining values of $\lambda$, for which there exist positive solutions of the nonlinear elliptic problem $-\Delta u=$ $\lambda a(x) f(u)$ in $\Omega, \partial u / \partial \mathbf{n}+b(x) g(u)=0$ on $\partial \Omega$. The proof of our main results is based upon unilateral global bifurcation theorem of López-Gómez.

\section{Introduction}

Let $\Omega$ be a bounded domain of Euclidean space $\mathbb{R}^{N}, N \geq 2$, with smooth boundary $\partial \Omega$. In this paper, we consider the nonlinear elliptic boundary value problem

$$
\begin{array}{cc}
-\Delta u=\lambda a(x) f(u) & \text { in } \Omega, \\
\frac{\partial u}{\partial \mathbf{n}}+b(x) g(u)=0 & \text { on } \partial \Omega,
\end{array}
$$

where $\Delta=\sum_{j=1}^{N}\left(\partial^{2} / \partial x_{j}^{2}\right), \lambda>0$ is a parameter, and $\mathbf{n}$ is the unit exterior normal to $\partial \Omega$.

We make the following assumptions.

(H0) $a \in C^{\theta}(\bar{\Omega})$ with $a(x)>0$ in $x \in \bar{\Omega} ; b \in C^{1+\theta}(\partial \Omega)$ with $b \geq 0$ and $b \neq \equiv$ on $\partial \Omega$.

(H1) $f \in C^{1}(\mathbb{R}, \mathbb{R})$ is an odd function with $f(s)>0$ for $s>0$ and there exist constants $f_{0}, f_{\infty} \in(0, \infty)$ and functions $\xi, h \in C^{1}(\mathbb{R}, \mathbb{R})$, such that

$$
\begin{array}{r}
f(s)=f_{0} s+\xi(s), \quad \xi(s)=o(|s|) \quad \text { as } s \longrightarrow 0, \\
f(s)=f_{\infty} s+h(s), \quad h(s)=o(|s|) \quad \text { as } s \longrightarrow+\infty .
\end{array}
$$

(H2) $g \in C^{1}(\mathbb{R}, \mathbb{R})$ is an odd function with $g(s)>0$ for $s>0$ and there exist constants $g_{0}, g_{\infty} \in(0, \infty)$ and functions $\zeta, k \in C^{1}(\mathbb{R}, \mathbb{R})$, such that

$$
\begin{gathered}
g(s)=g_{0} s+\zeta(s), \quad \zeta(s)=o(|s|) \quad \text { as } s \longrightarrow 0, \\
g(s)=g_{\infty} s+k(s), \quad k(s)=o(|s|) \quad \text { as } s \longrightarrow+\infty .
\end{gathered}
$$

A solution $u \in C^{2}(\bar{\Omega})$ of (1) is said to be positive if $u>0$ on $\bar{\Omega}$. The purpose of this paper is to study the global bifurcation of positive solutions for the asymptotically linear elliptic eigenvalue problems (1).

Let $X=C(\bar{\Omega})$ be the space of continuous functions on $\bar{\Omega}$. Then, it is a Banach space with the norm

$$
\|u\|=\max \{|u(x)| \mid x \in \bar{\Omega}\}
$$

Let

$$
P:=\{u \in X \mid u(x) \geq 0, x \in \bar{\Omega}\}
$$

Then $P$ is a cone which is normal and has a nonempty interior and $X=\overline{P-P}$. Moreover,

$$
\text { int } P=\{u \in P: u(x)>0 \text { for } x \in \Omega\} \text {. }
$$


By a constant $\lambda_{1}^{\infty}$ we denote the first eigenvalue of the eigenvalue problem

$$
\begin{gathered}
-\Delta \varphi=\lambda a(x) f_{\infty} \varphi \quad \text { in } \Omega, \\
\frac{\partial \varphi}{\partial \mathbf{n}}+b(x) g_{\infty} \varphi=0 \quad \text { on } \partial \Omega .
\end{gathered}
$$

By a constant $\lambda_{1}^{0}$ we denote the first eigenvalue of the eigenvalue problem

$$
\begin{gathered}
-\Delta \varphi=\lambda a(x) f_{0} \varphi \quad \text { in } \Omega, \\
\frac{\partial \varphi}{\partial \mathbf{n}}+b(x) g_{0} \varphi=0 \quad \text { on } \partial \Omega .
\end{gathered}
$$

It is well known (cf. Krasnosel'skii [1]) that, for $v \in\{0, \infty\}, \lambda_{1}^{v}$ is positive and simple and that it is a unique eigenvalue with positive eigenfunctions $\varphi_{1}^{v} \in C^{2+\theta}(\bar{\Omega})$. In what follows, the positive eigenfunction $\varphi_{1}^{\nu}$ is normalized as $\left\|\varphi_{1}^{\nu}\right\|=1$.

Let $\mathcal{S}$ be the closure of the set $\{(\lambda, u) \in(0, \infty) \times C(\bar{\Omega}) \mid$ $(\lambda, u)$ is a positive solution of (1) in $\mathbb{R} \times C(\bar{\Omega})\}$.

Theorem 1. Let (HO)-(H2) hold. Then, there exists an unbounded, closed, and connected component $\mathfrak{S}^{+} \subset(0, \infty) \times$ $C(\bar{\Omega})$ in $\mathcal{S}$, which joins $\left(\lambda_{1}^{0}, 0\right)$ with $\left(\lambda_{1}^{\infty}, \infty\right)$.

Corollary 2. Let (HO)-(H2) hold. Assume that either

$$
\lambda_{1}^{0}<\lambda<\lambda_{1}^{\infty}
$$

or

$$
\lambda_{1}^{\infty}<\lambda<\lambda_{1}^{0}
$$

Then (1) has at least one positive solution.

Remark 3. Ambrosetti et al. [2] and Umezu [3, 4] only studied the bifurcation from infinity for nonlinear elliptic eigenvalue problems. Nonlinear eigenvalue problems of ordinary differential equations have been extensively studied by many authors via fixed point theorem in cones and bifurcations techniques; see Henderson and Wang [5] and $\mathrm{Ma}[6,7]$ and the references therein. Ma and Thompson [7] considered the two-point boundary value problem

$$
u^{\prime \prime}(t)+r \tilde{a}(t) \tilde{f}(u)=0, \quad u(0)=u(1)=0 .
$$

By using the well-known Rabinowitz global bifurcation theorem [8], they proved the following.

Theorem A (see [7, Theorem 1.1]). Assume that

$(\mathrm{A} 1) \tilde{a}:[0,1] \rightarrow[0, \infty)$ is continuous and $\tilde{a}(t) \not \equiv 0$ on any subinterval of $[0,1]$;

(A2) $\tilde{f} \in C(\mathbb{R}, \mathbb{R})$ with $s \tilde{f}(s)>0$ for $s \neq 0$;

(A3) there exist $\tilde{f}_{0}, \tilde{f}_{\infty} \in(0, \infty)$ such that $\tilde{f}_{0}=$ $\lim _{|s| \rightarrow 0}(\tilde{f}(s) / s)$ and $\tilde{f}_{\infty}=\lim _{|s| \rightarrow \infty}(\tilde{f}(s) / s) ;$

(A4) $\tilde{\lambda}_{k}$ is the kth-eigenvalue of $u^{\prime \prime}(t)+\lambda \widetilde{a}(t) u=0, u(0)=$ $u(1)=0$.
If either $\tilde{\lambda}_{k} / \tilde{f}_{\infty}<r<\tilde{\lambda}_{k} / \tilde{f}_{0}$ or $\tilde{\lambda}_{k} / \tilde{f}_{0}<r<\tilde{\lambda}_{k} / \tilde{f}_{\infty}$, then (12) has two solutions $u_{k}^{+}$and $u_{k}^{-}$such that $u_{k}^{+}$has exactly $k-1$ zero in $(0,1)$ and is positive near 0 , and $u_{k}^{-}$has exactly $k-1$ zero in $(0,1)$ and is negative near 0 .

Obviously, Corollary 2 is a higher dimensional analogue of Ma and Thompson [7, Theorem 1.1] with $k=1$.

Remark 4. Shi [9] studied the exact number of all nontrivial solutions for

$$
\begin{gathered}
-\Delta u=\lambda f(u) \quad \text { in } \Omega, \\
u=0 \quad \text { on } \partial \Omega,
\end{gathered}
$$

for $\lambda$ in certain parameter range. He proved the existence of global smooth branches of positive solutions by using the implicit function theorem under some further restrictions on $f$.

Remark 5. Nonlinear elliptic eigenvalue problems have been studied in $[4,10]$ via topological degree and global bifurcation techniques. The positone case $f(0) \equiv 0$ is considered in [10], which is extended to the semipositone case $f(0)<0$ in [4]. An emphasis is, in Theorem 1 and Corollary 2, no assumption imposed on the boundedness of the function $h$ in (3).

Remark 6. Precup [11] applied the Moser-Harnack inequality for nonnegative superharmonic functions to produce a suitable cone and developed fixed point theorem in cones of Krasnoselskii-type to discuss the existence and multiplicity of positive solutions to elliptic boundary value problems

$$
\begin{gathered}
\Delta u+f(u)=0 \quad \text { in } \Omega, \\
u(x)>0 \quad \text { in } \Omega, \\
u=0 \quad \text { on } \partial \Omega .
\end{gathered}
$$

The constant $A$ in $[11,(3.1)]$ and the constant $B$ in $[11,(3.2)]$ are not optimal so that [11, Theorem 3.1] is not sharp. However, (10) and (11) in Corollary 2 are optimal. In fact, for the function

$$
f^{*}(s)=\lambda_{1}^{0} s+\arctan \left(\frac{s^{2}}{1+s^{2}}\right),
$$

which satisfies $f_{0}^{*}=f_{\infty}^{*}=\lambda_{1}^{0}$, the elliptic problem

$$
\begin{gathered}
\Delta u+f^{*}(u)=0 \quad \text { in } \Omega, \\
\frac{\partial u}{\partial \mathbf{n}}+u=0 \quad \text { on } \partial \Omega,
\end{gathered}
$$

has no positive solution.

The rest of this paper is organized as follows. The proof of our main results is based upon the unilateral global bifurcation theorem of López-Gómez, which is different from the topological degree arguments used in $[2-4,10]$. So, in Section 2, we state a preliminary result based upon unilateral global bifurcation theorem of López-Gómez. In Section 3, we reduce (1) into a compact operator equation. Section 4 is devoted to the proof of Theorem 1 . 


\section{Unilateral Global Bifurcation Theorem of López-Gómez}

Let $U$ be a Banach space with the normal $\|\cdot\|$. Let $\mathscr{L}(U)$ stand for the space of linear continuous operators in $U$. Let $J=(a, b) \subset \mathbb{R}$. Let $\mathfrak{F}: R \times U \rightarrow U$ be a nonlinear operator of the form

$$
\mathfrak{F}(\lambda, u)=I_{U}-\lambda K+\mathfrak{N}(\lambda, u),
$$

where $\mathfrak{N}: J \times U \rightarrow U$ is a continuous operator compact on bounded sets such that

$$
\mathfrak{N}(\lambda, u)=\circ(\|u\|),
$$

as $u \rightarrow 0$ uniformly in any compact interval of $J, K \in$ $\mathscr{L}(U)$ is a linear compact operator, and $r_{0} \neq 0$ is a simple characteristic value of $K$; that is,

$$
\operatorname{ker}\left[I_{U}-r_{0} K\right]=\operatorname{span}\left[\varphi_{0}\right],
$$

for some $\varphi_{0} \in U \backslash\{0\}$ satisfying

$$
\varphi_{0} \notin R\left[I_{U}-r_{0} K\right] .
$$

Let $\mathcal{S}$ be the closure of the set

$$
\{(\lambda, u) \mid(\lambda, u) \in J \times U, \mathfrak{F}(\lambda, u)=0, u \neq 0\} .
$$

Let $\mathfrak{C}^{+}$(resp., $\mathfrak{S}^{-}$) be the component of $\mathcal{S}$ that meets $\left(r_{0}, 0\right)$ and around $\left(r_{0}, 0\right)$ lies in $\mathcal{S} \backslash Q_{\epsilon, \eta}^{-}$(resp., $\mathcal{S} \backslash Q_{\epsilon, \eta}^{+}$); see [12, Section 6.4] for the details.

Let

$$
\Sigma:=\left\{\lambda \in J: \operatorname{dim} \operatorname{ker}\left[I_{U}-\lambda K\right] \geq 1\right\} .
$$

Then we present the unilateral global bifurcation theorem of López-Gómez; see [12, Theorem 6.4.3].

Lemma 7 (see [12], unilateral global bifurcation of López-Gómez). Assume $\Sigma$ is discrete, $r_{0} \in \Sigma$ satisfies (19), and the index Ind $(0, \mathfrak{N}(\lambda))$ changes sign as $\lambda$ crosses $r_{0}$. Then, for $v \in\{+,-\}$, the component $\mathfrak{C}^{v}$ satisfies one of the following:

(i) $\mathfrak{C}^{v}$ is unbounded in $\mathbb{R} \times U$;

(ii) there exists $r_{1} \in \Sigma \backslash\left\{r_{0}\right\}$ such that $\left(r_{1}, 0\right) \in \mathfrak{C}^{\nu}$;

(iii) $\boldsymbol{\sigma}^{v}$ contains a point

$$
(\lambda, y) \in R \times(Y \backslash\{0\}),
$$

where $Y$ is the complement of $\operatorname{ker}\left[I_{U}-r_{0} K\right]$ in $U$.

\section{Reduction to a Compact Operator Equation}

To establish Theorem 1 we begin with the reduction of (1) to a suitable equation for compact operators. According to Gilbarg and Trudinger [13], let $\mathscr{K}_{\infty}: C^{\theta}(\bar{\Omega}) \rightarrow C^{2+\theta}(\bar{\Omega})$ be the resolvent of the linear boundary value problem

$$
\begin{gathered}
-\Delta u=\phi \quad \text { in } \Omega, \\
\frac{\partial u}{\partial \mathbf{n}}+b(x) g_{\infty} u=0 \quad \text { on } \partial \Omega .
\end{gathered}
$$

By Amann [14, Theorem 4.2], $\mathscr{K}_{\infty}$ is uniquely extended to a linear mapping of $C(\bar{\Omega})$ compactly into $C^{1}(\bar{\Omega})$ and it is strongly positive, meaning that $\mathscr{K}_{\infty} \phi>0$ on $\bar{\Omega}$ for any $\phi \in C(\bar{\Omega})$ with the condition that $\phi \geq 0$ and $\phi \neq \equiv$ on $\bar{\Omega}$.

Let $\mathscr{R}_{\infty}: C^{1+\theta}(\partial \Omega) \rightarrow C^{2+\theta}(\bar{\Omega})$ be the resolvent of the linear boundary value problem

$$
\begin{gathered}
-\Delta u=0 \quad \text { in } \Omega, \\
\frac{\partial u}{\partial \mathbf{n}}+b(x) g_{\infty} u=\psi \quad \text { on } \partial \Omega .
\end{gathered}
$$

According to Amann [15, Section 4], $\mathscr{R}_{\infty}$ is uniquely extended to a linear mapping of $C(\partial \Omega)$ compactly into $C(\bar{\Omega})$. By the standard regularity argument, problem (1) is equivalent to the operator equation

$$
u=\lambda \mathscr{K}_{\infty}[a f(u)]+\mathscr{R}_{\infty}[b \tau(-k(u))] \quad \text { in } C(\bar{\Omega}) .
$$

Here $\tau: C(\bar{\Omega}) \rightarrow C(\partial \Omega)$ is the usual trace operator.

Similarly, let $\mathscr{K}_{0}: C^{\theta}(\bar{\Omega}) \rightarrow C^{2+\theta}(\bar{\Omega})$ be the resolvent of the linear boundary value problem

$$
\begin{gathered}
-\Delta u=\phi \quad \text { in } \Omega, \\
\frac{\partial u}{\partial \mathbf{n}}+b(x) g_{0} u=0 \quad \text { on } \partial \Omega .
\end{gathered}
$$

Then $\mathscr{K}_{0}$ is uniquely extended to a linear mapping of $C(\bar{\Omega})$ compactly into $C^{1}(\bar{\Omega})$ and it is strongly positive.

Let $\mathscr{R}_{0}: C^{1+\theta}(\partial \Omega) \rightarrow C^{2+\theta}(\bar{\Omega})$ be the resolvent of the linear boundary value problem

$$
\begin{gathered}
-\Delta u=0 \quad \text { in } \Omega \\
\frac{\partial u}{\partial \mathbf{n}}+b(x) g_{0} u=\psi \quad \text { on } \partial \Omega .
\end{gathered}
$$

Then $\mathscr{R}_{0}$ is uniquely extended to a linear mapping of $C(\partial \Omega)$ compactly into $C(\bar{\Omega})$. Furthermore, (1) is equivalent to the operator equation

$$
u=\lambda \mathscr{K}_{0}[a f(u)]+\mathscr{R}_{0}[b \tau(-\zeta(u))] \quad \text { in } C(\bar{\Omega})
$$

\section{The Proof of Main Results}

Obviously, (H1) and (H2) imply that

$$
\begin{array}{ll}
\lim _{|s| \rightarrow 0} \frac{\xi(s)}{|s|}=0, & \lim _{|s| \rightarrow 0} \frac{\zeta(s)}{|s|}=0, \\
\lim _{|s| \rightarrow \infty} \frac{h(s)}{|s|}=0, & \lim _{|s| \rightarrow \infty} \frac{k(s)}{|s|}=0 .
\end{array}
$$

Let

$$
\begin{aligned}
& \tilde{h}(r)=\max \{|h(s)| \mid 0 \leq s \leq r\}, \\
& \widetilde{k}(r)=\max \{|k(s)| \mid 0 \leq s \leq r\} .
\end{aligned}
$$


Then $\widetilde{h}$ and $\widetilde{k}$ are nondecreasing and there exist $r_{n} \nearrow \infty$ such that

$$
\lim _{n \rightarrow \infty} \frac{\widetilde{h}\left(r_{n}\right)}{r_{n}}=\lim _{n \rightarrow \infty} \frac{\widetilde{k}\left(r_{n}\right)}{r_{n}}=0 .
$$

Indeed, for any $r>0$, there exists $s \leq r$ such that $\widetilde{h}(r) / r=$ $|h(s)| / r$. Additionally, if we assume $s \leq C$ for some $C>0$, then it follows that $\widetilde{h}(r) / r \leq C / r \rightarrow 0$ as $r \rightarrow \infty$. On the other hand, we assume that there exists $r_{n}$ such that $s_{n} \rightarrow \infty$; then, it follows from (31) that $\widetilde{h}\left(r_{n}\right) / r_{n} \leq\left|h\left(s_{n}\right)\right| / s_{n} \rightarrow 0$ as $j \rightarrow \infty$, as desired.

We consider

$$
u=\lambda \mathscr{K}_{0}\left[a\left(f_{0} u+\xi(u)\right)\right]+\mathscr{R}_{0}[b \tau(-\zeta(u))] \quad \text { in } C(\bar{\Omega})
$$

as a bifurcation problem from the trivial solution $u \equiv 0$.

Define $K: X \rightarrow X$

$$
K u(t):=\mathscr{K}_{0}\left[a f_{0} u\right](t) ;
$$

then, $K$ is a strongly positive linear operator on $X$. It is easy to verify that $K: P \rightarrow P$ is completely continuous. From [14, Theorem 3.2], it follows that

$$
r(K)=\left[\lambda_{1}^{0}\right]^{-1}
$$

Define $\mathfrak{N}:[0, \infty) \times X \rightarrow X$ by

$$
\mathfrak{N}(\lambda, u):=\lambda \mathscr{K}_{0}[a \xi(u)]+\mathscr{R}_{0}[b \tau(-\zeta(u))] \quad \text { in } C(\bar{\Omega}) ;
$$

then, we have from (30) that

$$
\|\mathfrak{N}(\lambda, u)\|=\circ(\|u\|),
$$

locally and uniformly in $\lambda$.

It is very easy to check that (34) enjoys the structural requirements for applying the unilateral global bifurcation theory of $[12$, Sections $6.4,6.5]$ (by a counter example of Dancer [16], the global unilateral theorem of Rabinowitz [8] is false as stated. So, it cannot be used). As the theorem of Crandall and Rabinowitz [17] is applied to get the local bifurcation to positive solutions from $\left(\lambda_{1}^{0}, 0\right)$, the algebraic multiplicity of Esquinas and López-Gómez [18] (see [19, Chapter 4]) equals 1 and, therefore, by [12, Theorem 5.6.2] or [19, Proposition 12.3.1], the local index of 0 as a fixed point of $I-\lambda \mathscr{K}_{0}$ changes sign as $\lambda$ crosses $\lambda_{1}^{0}$. Therefore, it follows from Lemma 7 that there exists the component $\mathfrak{C}^{+}$ that satisfies one of the following:

(i) $\mathfrak{C}^{+}$is unbounded in $\mathbb{R} \times U$;

(ii) there exists $\lambda^{*} \in(0, \infty)$ with $\lambda^{*} \neq \lambda_{1}^{0}$ and $\psi \in C(\bar{\Omega})$ which changes its sign on $\Omega$, such that

$$
\left[I-\lambda^{*} \mathscr{K}_{0}\right] \psi=0, \quad\left(\lambda^{*}, 0\right) \in \mathfrak{C}^{+}
$$

(iii) $\mathfrak{C}^{+}$contains a point

$$
(\lambda, y) \in R \times(Y \backslash\{0\}),
$$

where $Y$ is the complement of $\operatorname{ker}\left[I_{X}-\lambda_{1}^{0} \mathscr{K}_{0}(a(\cdot))\right]$ in $X$.

In what follows, we will show that the above Case (ii) and Case (iii) do not occur.

In fact, if $(\mu, y) \in \mathfrak{C}^{+}$is a nontrivial solution of (34), then $y$ satisfies the problem

$$
\begin{gathered}
-\Delta y=\mu a(x) f(y) \quad \text { in } \Omega, \\
\frac{\partial y}{\partial \mathbf{n}}+b(x) g(y)=0 \quad \text { on } \partial \Omega,
\end{gathered}
$$

that is to say, $y$ satisfies the linear problem

$$
\begin{array}{cc}
-\Delta y=\mu a(x) \hat{f}(x) y & \text { in } \Omega, \\
\frac{\partial y}{\partial \mathbf{n}}+b(x) \hat{g}(x) y=0 & \text { on } \partial \Omega,
\end{array}
$$

where

$$
\begin{aligned}
& \widehat{g}(x)= \begin{cases}\frac{g(y(x))}{y(x)}, & \text { as } y(x) \neq 0, \\
g_{0}, & \text { as } y(x)=0,\end{cases} \\
& \widehat{f}(x)= \begin{cases}\frac{f(y(x))}{y(x)}, & \text { as } y(x) \neq 0, \\
f_{0}, & \text { as } y(x)=0 .\end{cases}
\end{aligned}
$$

We claim that

$$
y \in \mathfrak{C}^{+}: y \neq 0 \Longrightarrow y \in \operatorname{int} P \cup \operatorname{int}(-P) .
$$

Suppose, on the contrary, that $(\mu, y) \in \mathfrak{C}^{+}$with $y \neq 0$ and $y \in \partial P$. Then, there exists a sequence $\left\{\left(\mu_{n}, u_{n}\right)\right\} \subset \mathfrak{V}^{+} \cap P$ with $u_{n}>0$ for $n \in \mathbb{N}$, such that

$$
\left(\mu_{n}, u_{n}\right) \longrightarrow(\mu, y), \quad \text { as } n \longrightarrow \infty,
$$

and consequently

$$
y(x) \geq 0, \quad x \in \bar{\Omega} .
$$

Combining this with the fact that $(\mu, y)$ is a nontrivial solution of (34) and using the strong maximum principle [20, Theorem 2.4] and (42), it concludes that

$$
y(x)>0, \quad x \in \bar{\Omega},
$$

contracting $y \in \partial P$.

Similarly, for $(\eta, y) \in \mathfrak{C}^{+}$with $y \neq 0$ and $y \in \partial(-P)$, we get the desired contradiction.

Therefore, the claim (44) is true.

Since $f$ and $g$ are odd in $\mathbb{R},(\lambda,-u)$ is a solution of (34) if and only if $(\lambda, u)$ is a solution of (34). Combining this and (44) and using the fact that the eigenfunctions corresponding to the eigenvalue $\lambda_{j}$ of the operator $K$ with $j \geq 2$ have 
to change its sign in $\Omega$, it concludes that Case (ii) and Case (iii) cannot occur. Therefore, there exists an unbounded connected subset $\mathfrak{C}^{+}$of the set

$$
\{(\lambda, u) \in(0, \infty) \times P: u=A(\lambda, u), u \in \operatorname{int} P\} \cup\left\{\left(\lambda_{1}^{0}, 0\right)\right\}
$$

such that $\left(\lambda_{1}^{0}, 0\right) \in \mathfrak{C}^{+}$.

Proof of Theorem 1. It is clear that any solution of (34) of the form $(\lambda, u)$ yields a solution $u$ of $(1)$. We will show that $\mathfrak{C}^{+}$ joins $\left(\lambda_{1}^{0}, 0\right)$ to $\left(\lambda_{1}^{\infty}, \infty\right)$.

Let $\left(\mu_{n}, y_{n}\right) \in \mathfrak{C}^{+}$satisfy

$$
\mu_{n}+\left\|y_{n}\right\| \longrightarrow \infty
$$

We note that $\mu_{n}>0$, for all $n \in \mathbb{N}$ since $u=0$, is the only solution of (34) (i.e., (26)) for $\lambda=0$.

In fact, suppose on the contrary that $u$ is a nontrivial solution of the problem

$$
\begin{gathered}
-\Delta u=0 \quad \text { in } \Omega ; \\
\frac{\partial u}{\partial \mathbf{n}}+b(x) g(u)=0 \quad \text { on } \partial \Omega ;
\end{gathered}
$$

then, $u$ satisfies the linear problem

$$
\begin{gathered}
-\Delta u=0 \quad \text { in } \Omega, \\
\frac{\partial u}{\partial \mathbf{n}}+\widetilde{b}(x) u=0 \quad \text { on } \partial \Omega,
\end{gathered}
$$

where $\tilde{b}(x)=b(x)(g(u) / u)$. Together with $(\mathrm{H} 2)$ and the results of Krasnosel'skii [1], it follows that $u \equiv 0$ in $\bar{\Omega}$, which is a contradiction. Therefore, (34) (i.e., (26)) with $\lambda=0$ has only trivial solution.

Case $1\left(\lambda_{1}^{\infty}<\lambda<\lambda_{1}^{0}\right)$. In this case, we show that

$$
\left(\lambda_{1}^{\infty}, \lambda_{1}^{0}\right) \subseteq\left(\lambda \in \mathbb{R} \mid \exists(\lambda, u) \in \mathfrak{C}^{+}\right) .
$$

We divide the proof into two steps.

Step 1 . We show that if there exists a constant number $M>0$ such that

$$
\mu_{n} \subset(0, M]
$$

then $\mathfrak{C}^{+}$joins $\left(\lambda_{1}^{0}, 0\right)$ to $\left(\lambda_{1}^{\infty}, \infty\right)$.

From (53), we have $\left\|y_{n}\right\| \rightarrow \infty$. We divide the equation

$$
\begin{array}{r}
y_{n}=\mu_{n} \mathscr{K}_{\infty}\left[a\left(f_{\infty} y_{n}+h\left(y_{n}\right)\right)\right]+\mathscr{R}_{\infty}\left[b \tau\left(-k\left(y_{n}\right)\right)\right] \\
\text { in } C(\bar{\Omega}),
\end{array}
$$

by $\left\|y_{n}\right\|$ and set $v_{n}=y_{n} /\left\|y_{n}\right\|$. Since $v_{n}$ is bounded in $X$, choosing a subsequence and relabeling if necessary, we see that $v_{n} \rightarrow \bar{v}$ for some $\bar{v} \in X$ with $\|\bar{v}\|=1$. Moreover, from (31) and the fact that $\tilde{h}$ and $\widetilde{k}$ are nondecreasing together with the assertion $\left\|y_{n}\right\| \rightarrow \infty$, there exists some $r_{n_{j}} \leq\left\|y_{n}\right\|$ such that $\tilde{h}\left(r_{n_{j}}\right) \geq \tilde{h}\left\|y_{n}\right\|$ and $r_{n_{j}} \rightarrow \infty$. It follows that $\widetilde{h}\left(\left\|y_{n}\right\|\right) /\left\|y_{n}\right\| \leq \widetilde{h}\left(r_{n_{j}}\right) / r_{n_{j}} \rightarrow 0$ as $n \rightarrow \infty$. Subsequently, we have

$$
\lim _{n \rightarrow \infty} \frac{\left|h\left(y_{n}(t)\right)\right|}{\left\|y_{n}\right\|}=\lim _{n \rightarrow \infty} \frac{\left|k\left(y_{n}(t)\right)\right|}{\left\|y_{n}\right\|}=0,
$$

since $\left|h\left(y_{n}(t)\right)\right| /\left\|y_{n}\right\| \leq \widetilde{h}\left(\left|y_{n}(t)\right|\right) /\left\|y_{n}\right\| \leq \widetilde{h}\left(\left\|y_{n}\right\|\right) /\left\|y_{n}\right\|$ and $\left|k\left(y_{n}(t)\right)\right| /\left\|y_{n}\right\| \leq \widetilde{k}\left(\left|y_{n}(t)\right|\right) /\left\|y_{n}\right\| \leq \widetilde{k}\left(\left\|y_{n}\right\|\right) /\left\|y_{n}\right\|$. Therefore,

$$
\bar{v}=\bar{\mu} \mathscr{K}_{\infty}\left[a f_{\infty} \bar{v}\right] \quad \text { in } C(\bar{\Omega}),
$$

where $\bar{\mu}:=\lim _{n \rightarrow \infty} \mu_{n}$, again choosing a subsequence and relabeling if necessary.

Thus

$$
\begin{array}{cc}
-\Delta \bar{v}=\bar{\mu} a(x) f_{\infty} \bar{v} & \text { in } \Omega, \\
\frac{\partial \bar{v}}{\partial \mathbf{n}}+b(x) g_{\infty} \bar{v}=0 & \text { on } \partial \Omega .
\end{array}
$$

Since $\|\bar{v}\|=1$ and $\bar{v} \geq 0$, the strong positivity of $\mathscr{K}_{\infty}$ ensures that

$$
\bar{v}>0 \text { on } \bar{\Omega} \text {. }
$$

Thus $\bar{\mu}=\lambda_{1}^{\infty}$, and accordingly, $\mathscr{C}$ joins $\left(\lambda_{1}^{0}, 0\right)$ to $\left(\lambda_{1}^{\infty}, \infty\right)$.

Step 2. We show that there exists a constant $M$ such that $\mu_{n} \in$ $(0, M]$, for all $n$.

From $(\mathrm{H} 1)$ and $(\mathrm{H} 2)$, there exist constants $\alpha, \beta \in(0, \infty)$ such that

$$
\begin{array}{ll}
f(u) \geq \alpha u, & u \in[0, \infty), \\
g(u) \leq \beta u, & u \in[0, \infty) .
\end{array}
$$

By the same method to define $\mathscr{K}_{\infty}$ and $\mathscr{R}_{\infty}$ in Section 3, we may define $\mathscr{K}^{*}$ and $\mathscr{R}^{*}$ as follows.

Let $\mathscr{K}^{*}: C^{\theta}(\bar{\Omega}) \rightarrow C^{2+\theta}(\bar{\Omega})$ be the resolvent of the linear boundary value problem

$$
\begin{gathered}
-\Delta u=\phi \quad \text { in } \Omega, \\
\frac{\partial u}{\partial \mathbf{n}}+b(x) \beta u=0 \quad \text { on } \partial \Omega .
\end{gathered}
$$

Then, $\mathscr{K}^{*}$ is uniquely extended to a linear mapping of $C(\bar{\Omega})$ compactly into $C^{1}(\bar{\Omega})$ and it is strongly positive. Let $\mathscr{R}^{*}$ : $C^{1+\theta}(\partial \Omega) \rightarrow C^{2+\theta}(\bar{\Omega})$ be the resolvent of the linear boundary value problem

$$
\begin{gathered}
-\Delta u=0 \quad \text { in } \Omega, \\
\frac{\partial u}{\partial \mathbf{n}}+b(x) \beta u=\psi \quad \text { on } \partial \Omega .
\end{gathered}
$$

Let $\lambda_{1}^{*}$ be the eigenvalue of the linear problem

$$
\begin{gathered}
-\Delta u=\lambda a(x) \alpha u \quad \text { in } \Omega, \\
\frac{\partial u}{\partial \mathbf{n}}+b(x) \beta u=0 \quad \text { on } \partial \Omega,
\end{gathered}
$$


and let $\psi^{*} \in P$ be the corresponding eigenfunction. Then

$$
\begin{gathered}
-\Delta \psi^{*}=\lambda_{1}^{*} a(x) \alpha \psi^{*} \quad \text { in } \Omega, \\
\frac{\partial \psi^{*}}{\partial \mathbf{n}}+b(x) \beta \psi^{*}=0 \quad \text { on } \partial \Omega .
\end{gathered}
$$

Since $\left(\mu_{n}, y_{n}\right) \in \mathfrak{C}^{+}$satisfies (1) and $\left(\lambda_{1}^{*}, \psi^{*}\right)$ satisfies (63), it follows from (59) and Green's formula that

$$
\begin{aligned}
\left(\mu_{n}-\lambda_{1}^{*}\right) \int_{\Omega} a(x) \alpha \psi^{*}(x) u_{n}(x) d x \\
\leq \mu_{n} \int_{\Omega} a(x) f\left(u_{n}(x)\right) \psi^{*}(x) d x \\
\quad-\lambda_{1}^{*} \int_{\Omega} a(x) \alpha \psi^{*}(x) u_{n}(x) d x \\
=\int_{\Omega} \Delta \psi^{*}(x) u_{n}(x) d x-\int_{\Omega} \Delta u_{n}(x) \psi^{*}(x) d x \\
=\int_{\partial \Omega} u_{n} \frac{\partial \psi^{*}}{\partial \mathbf{n}} d \sigma-\int_{\partial \Omega} \psi^{*} \frac{\partial u_{n}}{\partial \mathbf{n}} d \sigma \\
=-\int_{\partial \Omega} b(x) \beta \psi^{*} u_{n} d \sigma+\int_{\partial \Omega} b(x) g\left(u_{n}\right) \psi^{*} d \sigma \\
\leq \int_{\partial \Omega} b(x)\left[\beta u_{n} \psi^{*}-\beta \psi^{*} u_{n}\right] d \sigma=0,
\end{aligned}
$$

and here $d \sigma$ is the surface element of $\partial \Omega$. Subsequently, $\mu_{n} \leq$ $\lambda_{1}^{*}$. Therefore, the component $\mathfrak{\sigma}^{+}$joins $\left(\lambda_{1}^{0}, 0\right)$ to $\left(\lambda_{1}^{\infty}, \infty\right)$.

Case $2\left(\lambda_{1}^{0}<\lambda<\lambda_{1}^{\infty}\right)$. In this case, if $\left(\mu_{n}, y_{n}\right) \in \mathfrak{C}^{+}$is such that

$$
\begin{gathered}
\lim _{n \rightarrow \infty}\left(\mu_{n}+\left\|y_{n}\right\|\right)=\infty, \\
\lim _{n \rightarrow \infty} \mu_{n}=\infty,
\end{gathered}
$$

then

$$
\left(\lambda_{1}^{0}, \lambda_{1}^{\infty}\right) \subseteq\left\{\lambda \in(0, \infty) \mid(\lambda, u) \in \mathfrak{C}^{+}\right\}
$$

and moreover,

$$
(\{\lambda\} \times X) \cap \mathfrak{C}^{+} \neq \emptyset
$$

Assume that there exists $M>0$, such that, for all $n \in \mathbb{N}$,

$$
\mu_{n} \in(0, M]
$$

Applying a similar argument to that used in Step 1 of Case 1 , after taking a subsequence and relabeling if necessary, it follows that

$$
\left(\mu_{n}, y_{n}\right) \longrightarrow\left(\lambda_{1}^{\infty}, \infty\right), \quad n \longrightarrow \infty
$$

Again $\mathfrak{S}^{+}$joins $\left(\lambda_{1}^{0}, 0\right)$ to $\left(\lambda_{1}^{\infty}, \infty\right)$ and the result follows.

Proof of Corollary 2. It is a directly desired consequence of Theorem 1.

\section{Conflict of Interests}

The authors declare that they have no competing interests regarding the publication of this paper.

\section{Acknowledgments}

The authors are very grateful to the anonymous referees for their valuable suggestions. This work was supported by the NSFC (no. 11361054 and no. 11201378), SRFDP (no. 20126203110004), and Gansu provincial National Science Foundation of China (no. 1208RJZA258).

\section{References}

[1] M. A. Krasnosel'skii, Positive Solutions of Operator Equations, P. Noordhoff, Groningen, The Netherlands, 1964.

[2] A. Ambrosetti, D. Arcoya, and B. Buffoni, "Positive solutions for some semi-positone problems via bifurcation theory," Differential and Integral Equations, vol. 7, no. 3-4, pp. 655-663, 1994.

[3] K. Umezu, "Global positive solution branches of positone problems with nonlinear boundary conditions," Differential and Integral Equations, vol. 13, no. 4-6, pp. 669-686, 2000.

[4] K. Umezu, "Bifurcation from infinity for asymptotically linear elliptic eigenvalue problems," Journal of Mathematical Analysis and Applications, vol. 267, no. 2, pp. 651-664, 2002.

[5] J. Henderson and H. Wang, "Positive solutions for nonlinear eigenvalue problems," Journal of Mathematical Analysis and Applications, vol. 208, no. 1, pp. 252-259, 1997.

[6] R. Ma, "Existence of positive solutions of a fourth-order boundary value problem," Applied Mathematics and Computation, vol. 168, no. 2, pp. 1219-1231, 2005.

[7] R. Ma and B. Thompson, "Nodal solutions for nonlinear eigenvalue problems," Nonlinear Analysis. Theory, Methods \& Applications, vol. 59, no. 5, pp. 707-718, 2004.

[8] P. H. Rabinowitz, "Some global results for nonlinear eigenvalue problems," Journal of Functional Analysis, vol. 7, pp. 487-513, 1971.

[9] J. Shi, "Exact multiplicity of solutions to superlinear and sublinear problems," Nonlinear Analysis. Theory, Methods \& Applications, vol. 50, no. 5, pp. 665-687, 2002.

[10] A. Ambrosetti and P. Hess, "Positive solutions of asymptotically linear elliptic eigenvalue problems," Journal of Mathematical Analysis and Applications, vol. 73, no. 2, pp. 411-422, 1980.

[11] R. Precup, "Moser-Harnack inequality, Krasnosel'skii type fixed point theorems in cones and elliptic problems," Topological Methods in Nonlinear Analysis, vol. 40, no. 2, pp. 301-313, 2012.

[12] J. López-Gómez, Spectral Theory and Nonlinear Functional Analysis, vol. 426 of CRC Research Notes in Mathematics, Chapman \& Hall, Boca Raton, Fla, USA, 2001.

[13] D. Gilbarg and N. S. Trudinger, Elliptic Partial Differential Equations of Second Order, Springer, New York, NY, USA, 1983.

[14] H. Amann, "Fixed point equations and nonlinear eigenvalue problems in ordered Banach spaces," SIAM Review, vol. 18, no. 4, pp. 620-709, 1976.

[15] H. Amann, "Nonlinear elliptic equations with nonlinear boundary conditions," in New Developments in Differential Equations, W. Eckhaus, Ed., vol. 21 of Mathematical Studies, pp. 43-63, North-Holland, Amsterdam, The Netherlands, 1976. 
[16] E. N. Dancer, "Bifurcation from simple eigenvalues and eigenvalues of geometric multiplicity one," The Bulletin of the London Mathematical Society, vol. 34, no. 5, pp. 533-538, 2002.

[17] M. G. Crandall and P. H. Rabinowitz, "Bifurcation from simple eigenvalues," Journal of Functional Analysis, vol. 8, pp. 321-340, 1971.

[18] J. Esquinas and J. López-Gómez, "Optimal multiplicity in local bifurcation theory. I. Generalized generic eigenvalues," Journal of Differential Equations, vol. 71, no. 1, pp. 72-92, 1988.

[19] J. López-Gómez and C. Mora-Corral, Algebraic Multiplicity of Eigenvalues of Linear Operators, vol. 177 of Operator Theory: Advances and Applications, Birkhäuser, Basel, Switzerland, 2007.

[20] Y. H. Du, Order Structure and Topological Methods in Nonlinear PDEs, vol. 1: Maximum Principles and Applications of Series on Partial Differential Equations and Applications, World Scientific, Singapore, 2006. 


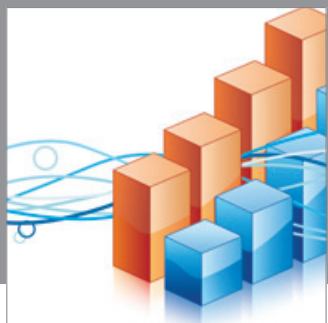

Advances in

Operations Research

mansans

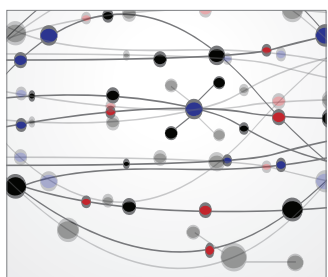

The Scientific World Journal
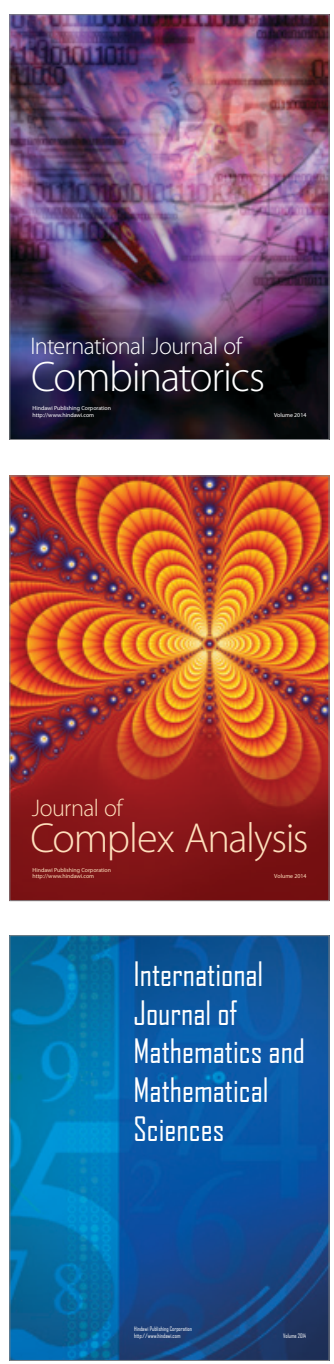
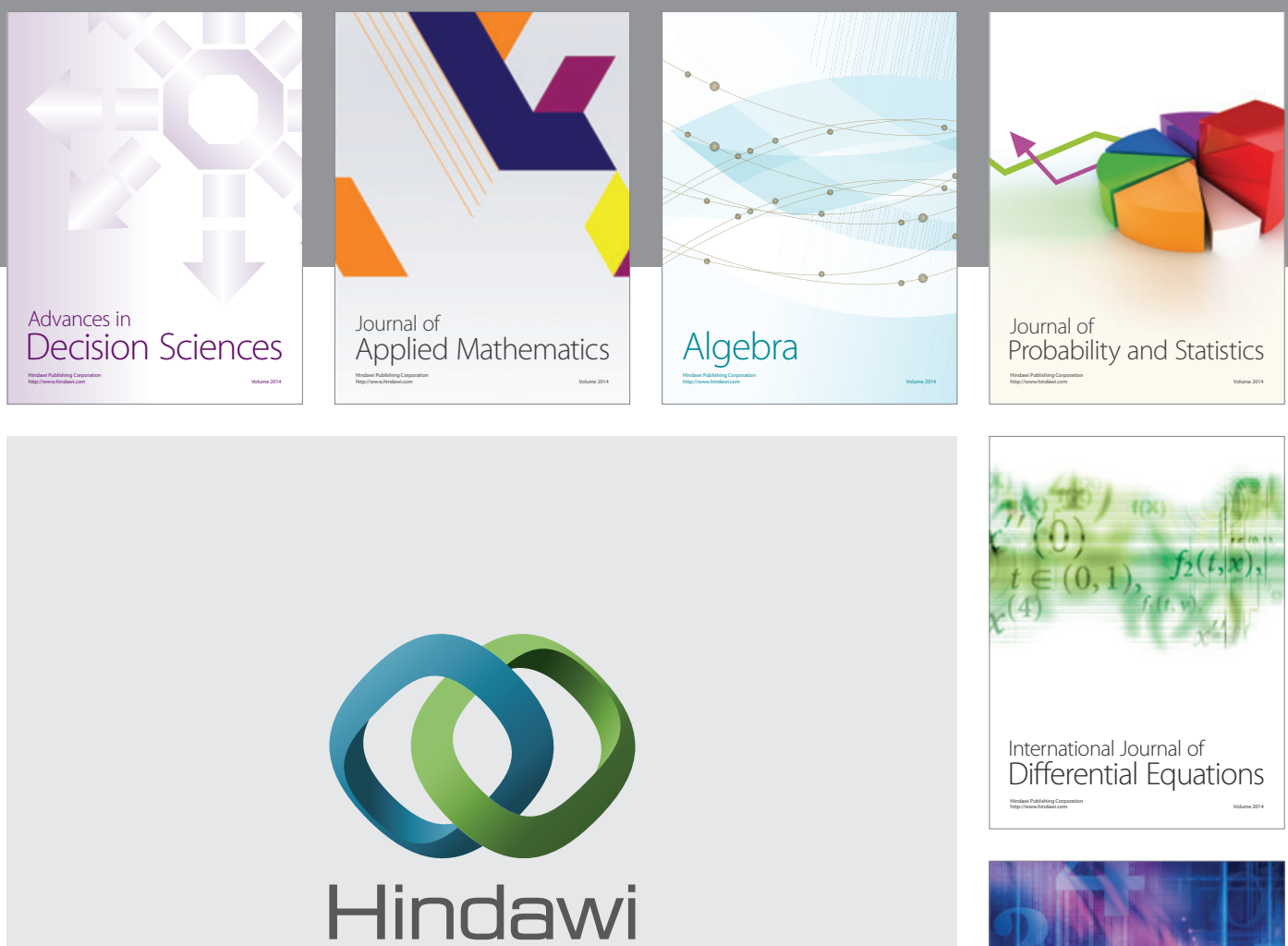

Submit your manuscripts at http://www.hindawi.com
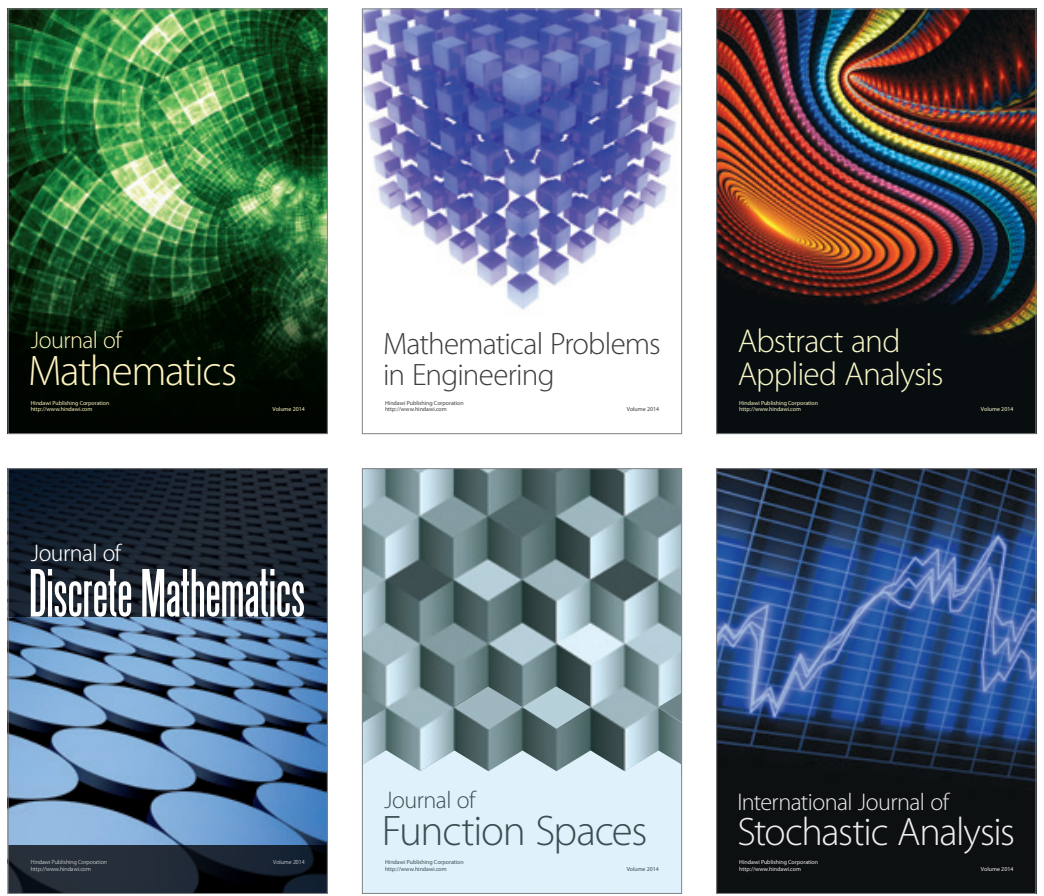

Journal of

Function Spaces

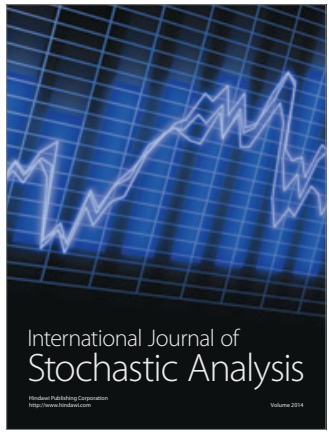

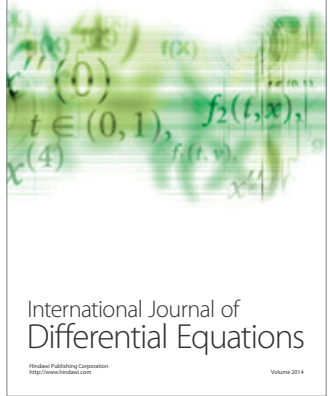
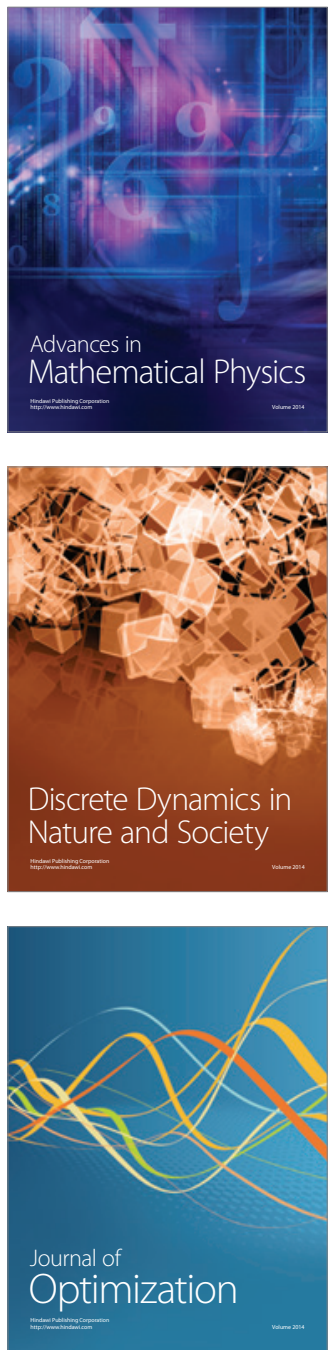